\title{
Las fracturas vertebrales y de cadera se asocian a un aumento de la mortalidad
}

Hip and vertebral fractures are associated with increased mortality

\author{
Objetivo \\ Evaluar la relación entre incidencia de fracturas y mortalidad a \\ largo plazo.
}

\section{Diseño y lugar}

Estudio de cohortes prospectivo de base poblacional observacional* realizado en nueve centros urbanos en Canadá.

\section{Pacientes}

Se seleccionó una muestra aleatoria de 7753 personas $(2187$ hombres y 5566 mujeres) mayores de 50 años (incluyendo áreas urbanas y rurales).

\section{Evaluación de los factores pronósticos}

Las fracturas se documentaron por un autoreporte validado mediante el contacto telefónico con el médico de cabecera $u$ hospital de referencia. Se agruparon según tipo de fractura: vertebral $(\mathrm{FV})$, cadera (FC), antebrazo, pelvis, muñeca, costillas, y "otras". Otras variables incluidas fueron las características antropométricas y demográficas, el uso de medicaciones, el estado previo a la fractura, los antecedentes de fractura previa, los hábitos de vida (alimentación, suplementos de calcio y vitamina $\mathrm{D}$, consumo de alcohol, actividad física regular y tabaquismo) y la calidad de vida (cuestionario SF-36).

\section{Medición de los resultados principales}

Se constató la muerte durante el seguimiento mediante contacto telefónico con los familiares; y para determinar la relación
Ioannidis G y col. CMAJ. 2009;181:265-71

independiente entre la muerte y el tipo de fractura se realizó un análisis multivariado de Cox*.

\section{Resultados principales}

Todos los tipos de fractura fueron más frecuentes en las mujeres a excepción de las de costilla. El riesgo de muerte luego de una FC fue igual en ambos sexos (23,5\%); mientras que para las FV fue algo superior en hombres (18,2\% vs. $15,7 \%)$.

Entre las mujeres, el riesgo de muerte fue mayor para aquellas con un FV durante el primer año (HR ajustado 3,7; IC95\% 1,1 a 12,8 ) y durante el segundo año de seguimiento (HR ajustado 3,2 ; IC95\% 1,2 a 8,1); seguido por el riesgo de muerte luego de una FC al año de seguimiento de (HR ajustado 3.0; IC95\% 1,0 a 8,7).

Otros variables predictoras de muerte en el análisis ajustado fueron la edad, la falta de educación universitaria, el sexo femenino, tener cuatro o más enfermedades crónicas, la falta de actividad física regular, el tabaquismo, el consumo de más de $100 \mathrm{mg} /$ día de cafeína y un bajo puntaje de calidad de vida.

\section{Conclusiones}

El antecedente de fracturas vertebral y/o de cadera se asocia a un mayor riesgo de muerte independientemente de otros factores conocidos.

Palabras claves: fracturas, mortalidad, factores predictores, fractura de cadera, fractura vertebral.

Keywords: fractures, mortality, predictors, hip fracture, vertebral fracture. Fuentes de financiamiento: no referida.

\section{Comentario}

Este estudio demuestra fuertemente la asociación independiente entre el antecedente de fracturas y el riesgo de muerte en mayores de 50 años. Contrariamente con otros estudios que habían demostrado mayor mortalidad en hombres ${ }^{1,2}$, este trabajo no encontró diferencias de mortalidad entre ambos sexos; y como era de esperar, otros factores como la edad, el tabaquismo, las enfermedades previas, la falta de actividad física regular y la educación universitaria tuvieron impacto sobre la mortalidad. Sin embargo, presenta algunas limitaciones, entre ellas el probable subregistro de fracturas vertebrales -ya que solo fueron registradas las fracturas que tuvieron alguna manifestación clínica- y la falta de discriminación del mecanismo de caída para diferenciar aquellas causadas por fragilidad ósea.

Como la mayoría de los trabajos que habían relacionado fracturas de cadera con mortalidad lo habían hecho sobre muestras pequeñas, estos sólo pudieron evaluar los riesgos asocia- dos a un solo tipo de fractura o estuvieron restringidos a ciertas áreas geográficas ${ }^{3,4,5}$. Por el contrario, este estudio aporta resultados con mayor validez externa ya que además del gran tamaño muestral, su población fue ambulatoria y había sido seleccionada al azar a nivel poblacional.

\section{Conclusiones de la comentadora}

La fuerte asociación entre el antecedente de fractura vertebral y de cadera y el aumento de la mortalidad durante los primeros años posteriores al evento refuerzan la necesidad de implementar estrategias durante este período para mejorar el pronóstico en este grupo de pacientes, así como la importancia de estrategias para prevenirlas.

Natalia Pace [ Servicio de Medicina Familiar y Preventiva. Área Programas Médicos del Plan de Salud. Hospital Italiano de Buenos Aires. natalia.pace@ hospitalitaliano.org.ar ]

Pace N. La fractura vertebral y de cadera se asocian a mayor mortalidad. Evid. actual. práct. ambul; 13 (2):54, Abr-Jun.2010. Comentado de loannidis $\mathrm{G}$ y col. Relation between fractures and mortality: results from the Canadian Multicentre Osteoporosis Study. CMAJ. 2009;181: 265-71. PMID: 19654194.

\section{Referencias}

1. Alegre-Lopez J y col. Factors associated with mortality and functional disability after hip fracture: an inception cohort study. Osteoporos Int 2005;16:729-36.

2. Magaziner Jet al. Predictors of functional recovery one year following hospital discharge for hip fracture: a prospective study. J Gerontol 1990;45:M101-7.

3. Center JR y col. Mortality after all major types of osteoporotic fractures in men and women: an observational study. Lancet 1999;353:878-82.

4. Katelaris AG y col/Health status before and mortality after hip fracture. Am J Public Health 1996;86:557-60.

5. Johnell O y col.. Mortality after osteoporotic fractures. Osteoporos Int 2004;15:38-42 